\title{
Research on hybrid SHEPWM based on different switching patterns
}

Tao Jing, Alexander Maklakov, Andrey Radionov, Sergei Baskov, Aleksandra Kulmukhametova

Department of Mechatronics and Automation, South Ural State University, Russia

\begin{tabular}{l}
\hline \hline Article Info \\
\hline Article history: \\
Received Mar 7, 2019 \\
Revised Apr 14, 2019 \\
Accepted Apr 19, 2019 \\
\hline
\end{tabular}

Keywords:

AC-DC power converters DC-AC power converters Medium voltage Reactive power control Variable speed drives

\begin{abstract}
This paper presents a hybrid pulse width modulation (HPWM) strategy based on different switching patterns of selective harmonic elimination pulse width modulation (SHEPWM) for the three-level neutral point clamped (3L-NPC) converter. Specific low-order harmonics can be eliminated by SHEPWM at low switching frequency, while the remaining high-order harmonics can be selected to be simply filtered by additional hardware. Large oscillation waveform usually occurs in the transition instant between two diverse modulation situations, therefore switching between distinct switching patterns can be problematic if no effective means is taken, especially when the effect of smooth and fast transition at any time is considerable. A universal and valid control strategy, which maintains the high-quality output voltage and current, is proposed and implemented in this paper to address this issue. Simulation results obtained from MATLAB/SIMULINK are presented to analyze the performance and validate the feasibility and effectiveness of this control scheme.
\end{abstract}

Copyright $(2019$ Institute of Advanced Engineering and Science. All rights reserved.

\section{Corresponding Author:}

Tao Jing,

Departement of Mechatronics and Automation,

South Ural State University,

76 Lenin Pr. 454080, Chelyabinsk, Russia.

Email: jingtao19940214@gmail.com

\section{INTRODUCTION}

For the past few years, power converters have been developing towards the direction of high voltage and high power for purpose of improving the quality and efficiency of power equipments and systems. Due to the advantages of the three-level neutral point clamped (3L-NPC) converter, including high voltage capability, low dv/dt, low switching losses, good output quality and low electromagnetic compatibility (EMC) concerns, it has been used in many commercial and industrial applications, for instance, high voltage direct current (HVDC), high-voltage high-power AC variable speed drives and flexible ac transmission systems (FACTS) [1-4].

The pulse width modulation (PWM) control method is a fairly critical technique in the research of 3L-NPC converter, and plays a vital role in reducing system switching losses and improving power system efficiency. It determines not only whether the target of power conversion can be realized, but also the final output quality of the voltage and current. Hence, the performance characteristics of a power converter (e.g., voltage quality and power factor) greatly depend on the selection of the appropriate modulation strategy and modification of its operating parameters [5-8].

The low switching frequency should be specially paid attention to the high power applications, which is in charge of the high-efficiency of system as well as the reduction in switching loss [9]. Modulation methods like carrier-based sinusoidal pulse width modulation (CB-SPWM) and space vector pulse width modulation (SVPWM) are commonly not suitable for the applications where the switching frequency is below $1 \mathrm{kHz}$. The low-order harmonics will occur in the sideband near the carrier frequency, so that the high 
distortion will be produced in the output voltage and current. Nevertheless, selective harmonic elimination pulse width modulation (SHEPWM), which can be utilized to decrease the switching losses and further increase the reliability and efficiency of power converters while be utilized with good power quality in low switching frequency situations, as one of the preferred modulation strategies for high power converters [10 12]. This strategy can be realized by means of the solution for solving a set of nonlinear and transcendental equations, and typical methods have been dealt with in numerous papers [13-16].

The hybrid SHEPWM presented in this paper combines one switching pattern with another one. The main features of this modulation strategy are the smooth and rapid switching transition and the reduction in switching losses. The transition conditions can be regulated by changing the combination of two switching patterns. Furthermore, this hybrid SHEPWM is based on a versatile and effective algorithm, which is very convenient to implement and process. And it is a universal and comprehensive solution for different situations.

In this paper, the feasibility and effectiveness of the hybrid SHEPWM proposed is researched. The rest of the paper is organized as follows. Section II presents the establishment of SHEPWM mathematical model for 3L-NPC converter. Theoretical part of proposed hybrid SHEPWM modulation strategy and transition scheme are introduced in Section III. In Section IV, simulation results are analyzed and its smooth and fast transition is also put forward in this section. Finally the conclusions are given in Section V.

\section{ESTABLISHMENT OF SHEPWM MATHEMATICAL MODEL FOR 3L-NPC CONVERTER}

The Figure 1 shows the topology of 3L-NPC converter. The DC-bus voltage is divided into three levels $\left(V_{d c} / 2,0\right.$, and $\left.-V_{d c} / 2\right)$ as well as output phase voltage.

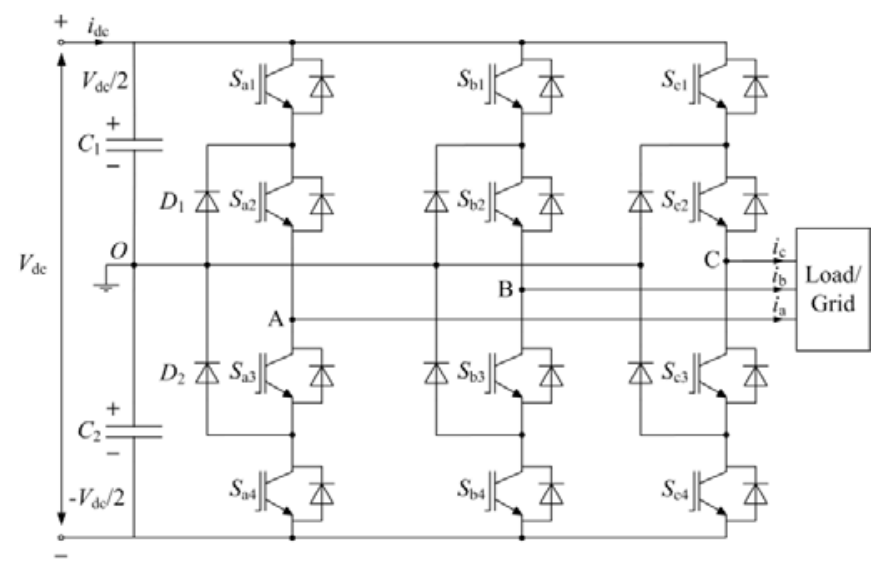

Figure 1. Topology of 3L-NPC converter.

The output phase-A voltage, for instance, can be expressed as:

$$
V_{A O}=\left\{\begin{array}{r}
V_{d c} / 2,\left(S_{a 1}, S_{a 2}\right)=1, \text { and },\left(S_{a 3}, S_{a 4}\right)=0 \\
0,\left(S_{a 2}, S_{a 3}\right)=1, \text { and },\left(S_{a 1}, S_{a 4}\right)=0 \\
-V_{d c} / 2,\left(S_{a 3}, S_{a 4}\right)=1, \text { and },\left(S_{a 1}, S_{a 2}\right)=0
\end{array}\right.
$$

Here, the main attention is focused on the output phase-A voltage $V_{A O}$. Through artificial arrangement of switching angles for SHEPWM, we can obtain the common output phase-A voltage waveforms of the 3L-NPC converter as shown in Figure 2. The number of eliminated harmonics is determined by the number of switching angles. 


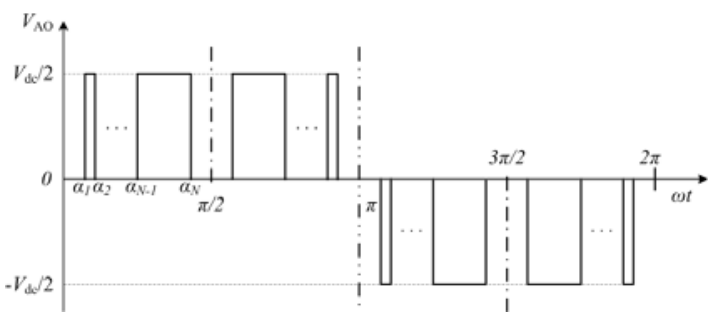

(a)

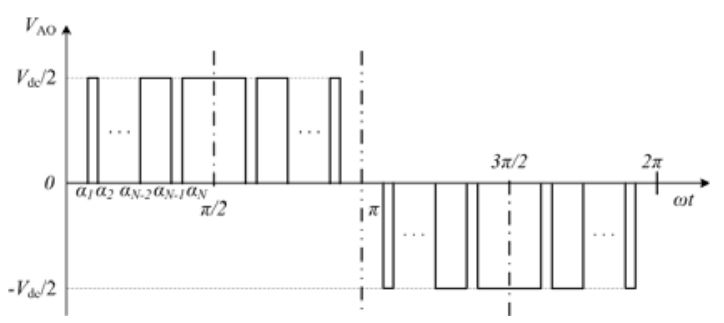

(b)

Figure 2. The common output phase-A voltage waveforms of the 3L-NPC converter.

(a) $N=$ even, (b) $N=$ odd.

According to Dirichlet theorem, the following Fourier series can be obtained for the above output phase-A voltage waveform:

$$
V_{A O}(\omega t)=\frac{A_{0}}{2}+\sum_{n=1}^{\infty}\left[A_{n} \sin (n \omega t)+B_{n} \cos (n \omega t)\right]
$$

where,

$$
\begin{aligned}
& A_{n}=\frac{1}{\pi} \int_{0}^{2 \pi} V_{A O}(\omega t) \sin (n \omega t) d(\omega t) \\
& B_{n}=\frac{1}{\pi} \int_{0}^{2 \pi} V_{A O}(\omega t) \cos (n \omega t) d(\omega t)
\end{aligned}
$$

The Fourier analysis of above output voltage waveform can be constrained to the first quarter-cycle because of the even quarter-wave symmetry and odd half-wave symmetry of the output voltage waveform, and hence only odd-order harmonics are existent without even-order harmonics.

$$
\begin{cases}A_{n}= \begin{cases}0, & n=\text { even } \\ \frac{4}{n \pi} \frac{V_{d c}}{2} \sum_{k=1}^{N}(-1)^{k+1} \cos \left(n \alpha_{k}\right), & n=\text { odd }\end{cases} \\ B_{n}=0\end{cases}
$$

where $N$ is the number of switching angles within the first quarter-cycle of the fundamental period, and $\alpha_{1}, \alpha_{2}, \ldots, \alpha_{N}$ which satisfy (6) below.

$$
0^{\circ}<\alpha_{1}<\alpha_{2}<\ldots<\alpha_{N}<90^{\circ}
$$

Here, the modulation index $M$ is defined as the radio of the fundamental amplitude of the output voltage $A_{1}$ divided by half of the $\mathrm{DC}$ voltage $V_{d c} / 2$, that is,

$$
M=\frac{A_{1}}{V_{d c} / 2}
$$

In the symmetric three-phase system, the triple-order harmonics can be cancelled by each other automatically, and the nontriple-order harmonics will be eliminated by selecting optimal switching angles $\alpha_{1}$, $\alpha_{2}, \ldots, \alpha_{N}$ through (8) below with modulation index $M$.

$$
\left\{\begin{array}{c}
A_{1}(\alpha)=\frac{4}{\pi} \sum_{k-1}^{N}(-1)^{k+1} \cos \left(\alpha_{k}\right)=M \\
A_{5}(\alpha)=\frac{4}{5 \pi} \sum_{k-1}^{N}(-1)^{k+1} \cos \left(5 \alpha_{k}\right)=0 \\
\mathrm{M} \\
A_{n}(\alpha)=\frac{4}{n \pi} \sum_{k-1}^{N}(-1)^{k+1} \cos \left(\alpha_{k}\right)=0
\end{array}\right.
$$


where $n$ is the highest order harmonic needed to be eliminated, and $\alpha=\left[\alpha_{1}, \alpha_{2}, \ldots, \alpha_{N}\right]$.

Obviously, this equation set is nonlinear and transcendental, and the switching angles can be conveniently solved by the fsolve function in the MATLAB in order to simplify the calculation procedure and take full advantage of the high computational performance of computer, therefore much computation effort can be done with ease. The final results of each switching angle for SHEPWM obtained are applied to control the output waveform with the elimination of specific low-order harmonics. Nonetheless, the selection of suitable initial values is crucial for the iterative convergence. According to reference [17], the initial values for $N$ switching angles (here, $N=$ odd) unipolar SHEPWM can be derived as

$$
\left\{\begin{array}{c}
\alpha_{2 k-1}^{0}=30^{\circ}+120^{\circ} \cdot k /(N+1)-\Delta \alpha \\
\alpha_{2 k}^{0}=30^{\circ}+120^{\circ} \cdot k /(N+1)+\Delta \alpha \\
\alpha_{N}^{0}=90^{\circ}-\Delta \alpha
\end{array}\right.
$$

where $k=1,2, \ldots,(N-1) / 2$, and $\Delta \alpha$ is an optimal small angle, which is used to avoid singularity of fsolve function. After test, $\Delta \alpha=0.3 \sim 0.5$ and some other values can ensure iterative convergence.

Finally, several programs are written based on M language in MATLAB to get various curves based on different numbers of switching angles and modulation index ranges from 0 to 1 . Here, $N=17$ and $N=19$ are taken as the examples, as shown in Figure 3.

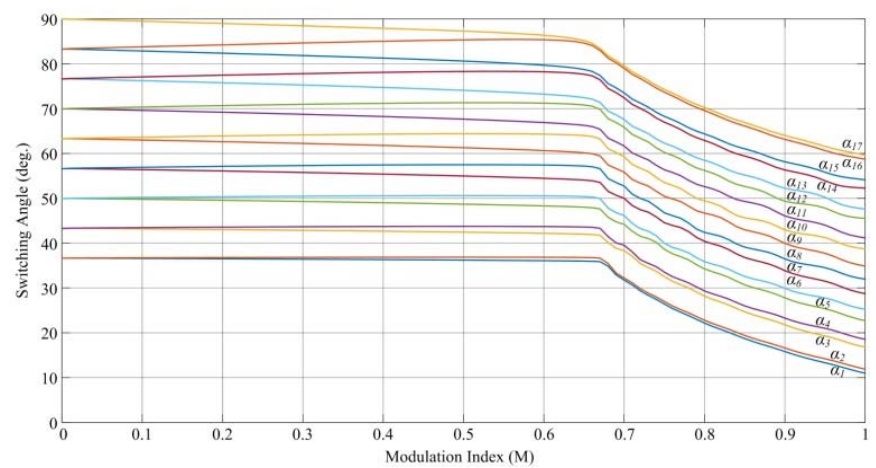

(a)

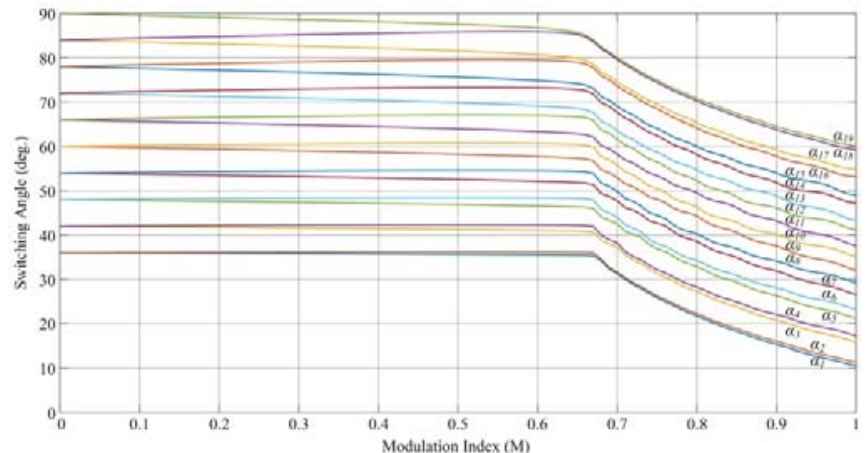

(b)

Figure 3. The relation curves between switching angle (deg.) and modulation index $(M)$ (a) $N=17$, (b) $N=19$.

\section{PROPOSED HYBRID SHEPWM MODULATION STRATEGY AND TRANSITION SCHEME}

\subsection{Proposed modulation strategy}

Generally, the maximum number of selected harmonics to be removed depends on the allowable switching frequency of semiconductor modules, which has the following relationship: 


$$
k=\frac{f_{s w . \max }}{2 \cdot f_{1}}-1=N-1
$$

where $k$ is the maximum number of selected harmonics to be removed, $f_{s w \text { max }}$ is the allowable switching frequency of semiconductor modules, and $f_{1}$ is the fundamental frequency.

According to (10), a universal modulation strategy for a power converter limited to a switching frequency of $450 \mathrm{~Hz}$ (due to the IGBT module) with different fundamental frequencies at $M=0 \sim 1$ is shown in Figure 4.

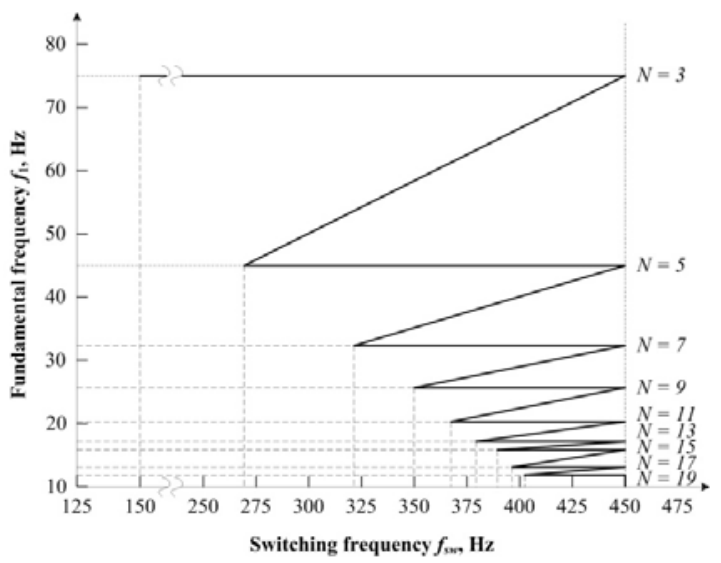

Figure 4. Modulation strategy using SHEPWM in the modulation region.

\subsection{Proposed transition scheme}

Every moment has its corresponding output state in the modulation process of SHEPWM, as shown in Figure 5. In order to illustrate its principle, several parameters are determined as follow: $N=19, M=0.85$ and $f_{1}=11.84 \mathrm{~Hz}$ (where, $\alpha_{1}=18.25^{\circ}, \alpha_{2}=18.84^{\circ}, \alpha_{3}=23.76^{\circ}, \alpha_{4}=24.90^{\circ}, \alpha_{5}=29.33^{\circ}, \alpha_{6}=30.94^{\circ}$, $\alpha_{7}=34.94^{\circ}, \quad \alpha_{8}=36.94^{\circ}, \quad \alpha_{9}=40.59^{\circ}, \quad \alpha_{10}=42.89^{\circ}, \quad \alpha_{11}=46.21^{\circ}, \quad \alpha_{12}=48.64^{\circ}, \quad \alpha_{13}=51.41^{\circ}$, $\alpha_{14}=54.64^{\circ}, \alpha_{15}=56.68^{\circ}, \alpha_{16}=60.67^{\circ}, \alpha_{17}=62.00^{\circ}, \alpha_{18}=66.73^{\circ}, \alpha_{19}=67.37^{\circ}$ ).

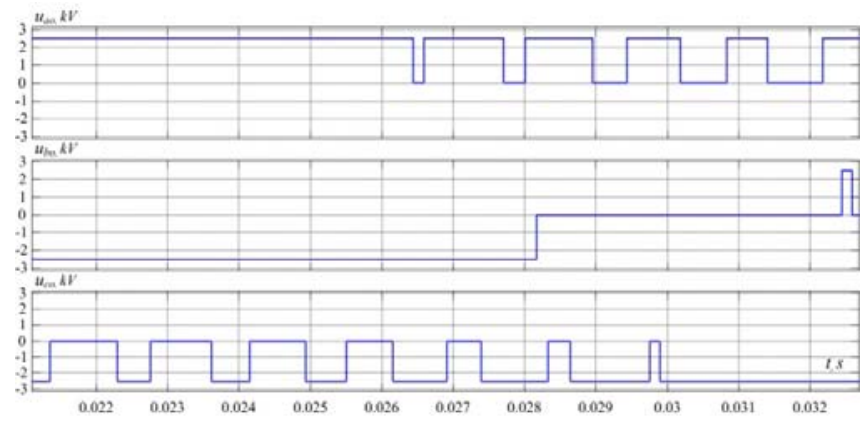

Figure 5. Phase voltage waveforms of the 3L-NPC converter during the modulation process of SHEPWM $(N=19)$.

The output phase voltage waveforms above, in a sense, can be regarded and expressed as the corresponding space vectors on the basis of space vector representation. In addition, the corresponding space vectors and their duty cycles from $90^{\circ}$ to $120.1^{\circ}$ are presented in Table 1 . 
Table 1. Corresponding space vectors of SHEPWM $(N=19)$ and their duty cycles from $90^{\circ}$ to $120.1^{\circ}$

\begin{tabular}{ccc}
\hline No. & Duty cycle & Space vector \\
\hline 1 & $90^{\circ}-91.02^{\circ}$ & {$[\mathrm{PNN}]$} \\
2 & $91.02^{\circ}-95.03^{\circ}$ & {$[\mathrm{PNO}]$} \\
3 & $95.03^{\circ}-97.03^{\circ}$ & {$[\mathrm{PNN}]$} \\
4 & $97.03^{\circ}-100.66^{\circ}$ & {$[\mathrm{PNO}]$} \\
5 & $100.66^{\circ}-102.96^{\circ}$ & {$[\mathrm{PNN}]$} \\
6 & $102.96^{\circ}-106.29^{\circ}$ & {$[\mathrm{PNO}]$} \\
7 & $106.29^{\circ}-108.72^{\circ}$ & {$[\mathrm{PNN}]$} \\
8 & $108.72^{\circ}-111.49^{\circ}$ & {$[\mathrm{PNO}]$} \\
9 & $111.49^{\circ}-112.72^{\circ}$ & {$[\mathrm{PNN}]$} \\
10 & $112.72^{\circ}-113.36^{\circ}$ & {$[\mathrm{ONN}]$} \\
11 & $113.36^{\circ}-114.73^{\circ}$ & {$[\mathrm{PNN}]$} \\
12 & $114.73^{\circ}-116.77^{\circ}$ & {$[\mathrm{PNO}]$} \\
13 & $116.77^{\circ}-118.10^{\circ}$ & {$[\mathrm{PNN}]$} \\
14 & $118.10^{\circ}-119.42^{\circ}$ & {$[\mathrm{ONN}]$} \\
15 & $119.42^{\circ}-120.10^{\circ}$ & {$[\mathrm{PNN}]$} \\
\hline
\end{tabular}

When the hybrid SHEPWM method is adopted, the switching problem between two different SHEPWM switching patterns should be necessarily considered. One of the objectives of transition is to switch smoothly from one state to another, so as to prevent the large impulse current produced at a transition instant and its adverse effects on electrical equipment or power grid. And another one is to switch quickly, so that the transition can satisfy the dynamic response demand of the power systems. In other words, smooth transition should be done in one switching period [18-20].

In order to realize the above goals, the following two conditions are generally required. It is necessary to ensure that the phase angle and amplitude of the fundamental phase voltage before and after transition are consistent. Otherwise, the sudden change of the fundamental phase voltage will occur, which will lead to overshoot of the output current.

The number of switching actions should not be more than one. If not, it will have some negative influence on the converter, such as greater switching losses, sudden changes in phase voltage and short pulses.

All of the above considerations are valid for the transition from previous SHEPWM switching pattern to the current one. The transition algorithm is implemented quite simply, and the main task is to design a "vector detector". This detector (see Figure 6) continuously detects the generated states $[\mathrm{P}]$, $[\mathrm{O}]$ and $[\mathrm{N}]$ between two switching patterns, and at the moment when the signal on whether to perform switching transition is on, the control system performs the transition if all previous conditions given are met.

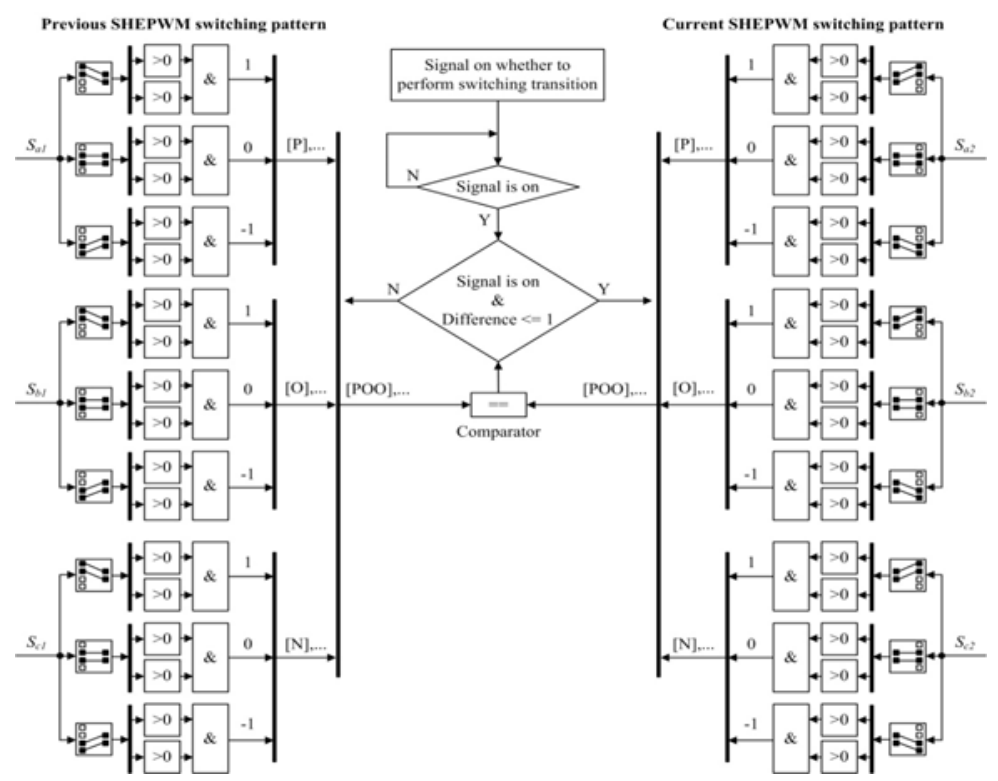

Figure 6. Block diagram of proposed hybrid SHEPWM transition scheme.

Int J Pow Elec \& Dri Syst Vol. 10, No. 4, Dec 2019 : 1875 - 1884 


\section{SIMULATION RESULTS AND ANALYSIS}

To verify and evaluate the states of output voltage and current waveforms acquired from 3L-NPC converter using the proposed transition scheme, the simulation study is implemented in MATLAB/SIMULINK platform. In our model, the default state of the signal on whether to perform switching transition is the running status.

Figure 7 shows simulation results of output phase-A voltage and current and output line voltage before and after transition from SHEPWM $N=19$ to SHEPWM $N=17$ in the case of applying the proposed transition scheme.

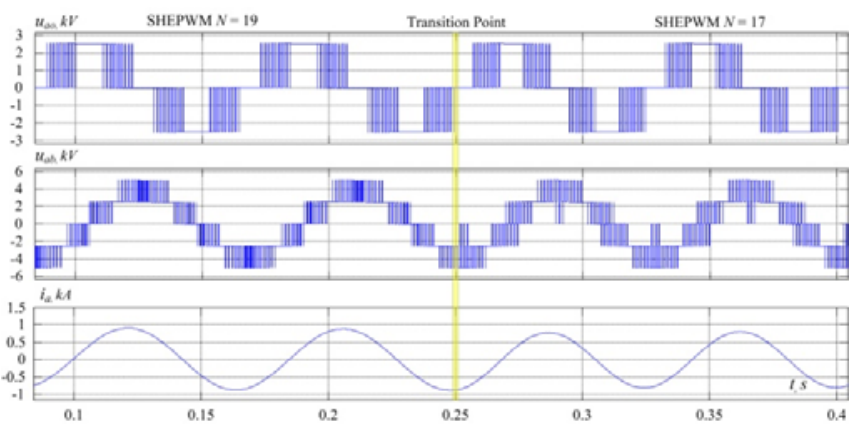

Figure 7. Simulation results of output phase-A voltage and current and output line voltage before and after transition from SHEPWM $N=19$ to $\operatorname{SHEPWM~} N=17$.

Here, $u_{a o}$ and $i_{a}$ represents output phase-A voltage and current, respectively, and $u_{a b}$ is output line voltage. The harmonic spectrums of $u_{a b}$ before and after transition are shown in Fig. 8. It is seen that the loworder harmonics below 50th-order of harmonic are both eliminated, which is the same as aforementioned theoretical analysis.

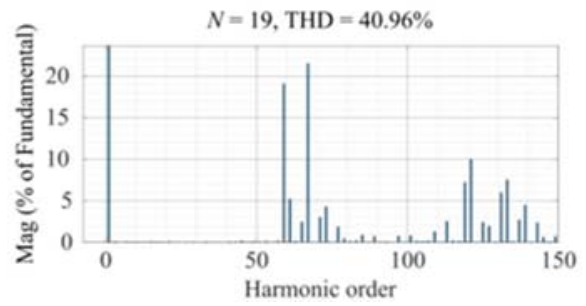

(a)

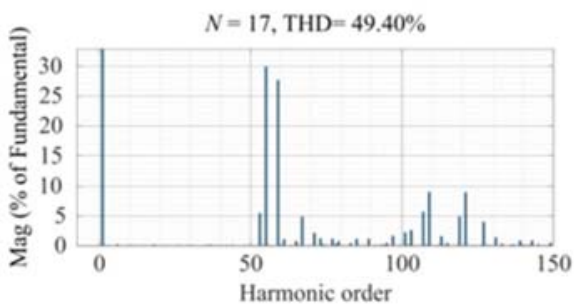

(b)

Figure 8. Simulation results of harmonic spectrums of $u_{a b}$ : a) SHEPWM $N=19$, b) SHEPWM $N=17$

Each output phase voltage before and after transition is shown in Figure 9. As can be seen from Figure 9 that the space vectors of the previous state and the latter state are the same, so that the transition can be smoothly performed during the switching process at time $t=0.25 \mathrm{~s}$. And this transition finishes in a short time. 


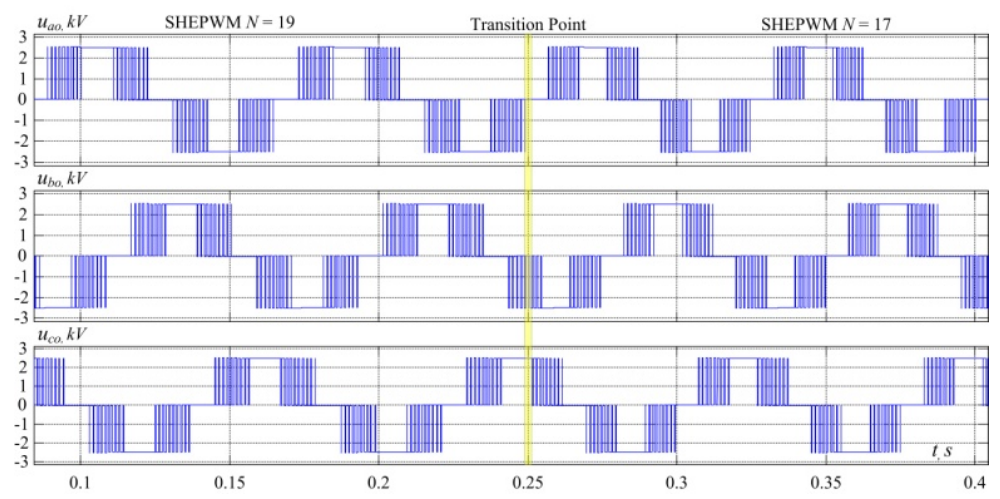

(a)

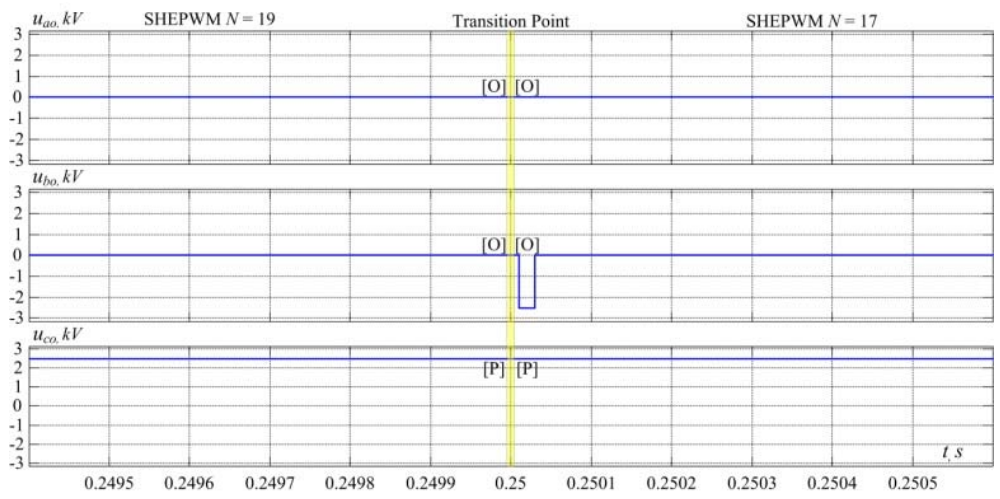

(b)

Figure 9. Simulation results of each output phase voltage before and after transition. (a) Transition from SHEPWM $N=19$ and SHEPWM $N=17$. (b) Zoom of transition point at time $t=0.25 \mathrm{~s}$

One of the significant indicators for the smooth transition between different SHEPWM switching patterns is that there is no sudden change in each output phase current during transition, including phase and amplitude of fundamental, and harmonics of currents. From Figure 10, we can easily find that simulation results of each output phase current $i_{a}, i_{b}$ and $i_{c}$ before and after transition achieve the previously mentioned target.

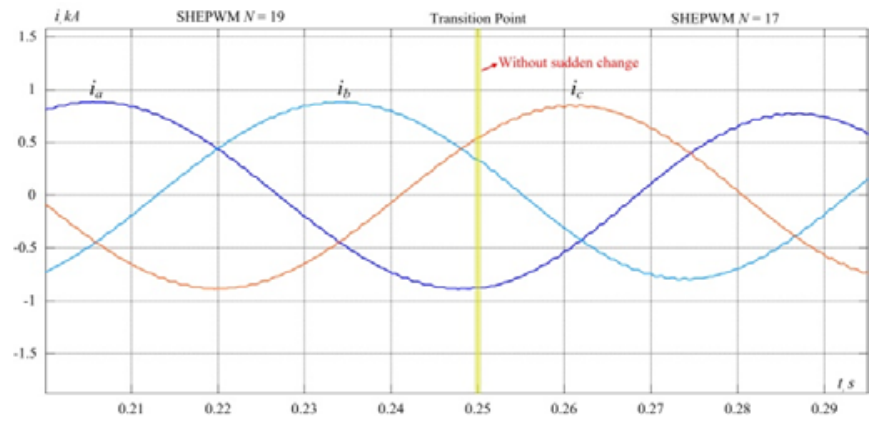

Figure 10. Simulation results and zoom of each output phase current $i_{a}, i_{b}$ and $i_{c}$ before and after transition.

In general, the transition from SHEPWM $N=19$ to SHEPWM $N=17$ is smooth and rapid, which means the proposed hybrid SHEPWM scheme for 3L-NPC converter is well realized. 


\section{CONCLUSION}

This paper proposed a smooth and quick transition scheme of a hybrid SHEPWM based on different switching patterns for 3L-NPC converter, and it can also be extended to be adopted for the transition between other different modulation strategies. This transition scheme has an extensive applicative prospect, such as high-power AC variable speed drives. It ensures that no additional switching counts occur, so that switching losses are reduced and output quality is improved. And the smooth transition can be completed within one switching period by properly setting the transition conditions. Finally, this scheme is verified by simulation, and further validation will be carried out with experimental equipment in the next research steps.

\section{ACKNOWLEDGEMENTS}

The work was supported by Act 211 Government of the Russian Federation, contract № 02.A03.21.0011.

\section{REFERENCES}

[1] J. Rodriguez, S. Bernet, P. Steimer, and I. Lizama, "A survey on neutral-point-clamped inverters," IEEE Transactions on Industrial Electronics, vol. 57(7), pp. 2219-2230, Jul 2010.

[2] H. Abu-Rub, J. Holtz, J. Rodriguez, and G. Baoming, "Medium-voltage multilevel converters-State of the art, challenges, and requirements in industrial applications," IEEE Transactions on Industrial Electronics, vol. 57(8), pp. 2581-2596, Aug 2010.

[3] B. Wu, et al., "High-power converters and AC drives," 2nd Edition, pp. 480, New York: Wiley, 2017.

[4] Y. Kucherov and S. Utts, "The FACTS and HVDC equipment application for reliability, flexibility and controllability increase in the metropolitan area power system," in 11th IET International Conference on AC and DC Power Transmission, pp. 1-6, Birmingham, 2015.

[5] H. S. Patel and R. G. Hoft, "Generalized techniques of harmonic elimination and voltage control in thyristor inverters: Part i-harmonic elimination," IEEE Transactions on Industry Applications, vol. IA-9(3), pp. 310-317, May 1973.

[6] W. Fei, X. Du and B. Wu, "A generalized half-wave symmetry SHE-PWM formulation for multilevel voltage inverters," IEEE Transactions on Industrial Electronics, vol. 57(9), pp. 3030-3038, Sept 2010.

[7] M. S. A. Dahidah, G. Konstantinou and V. G. Agelidis, "A review of multilevel selective harmonic elimination PWM: Formulations, solving algorithms, implementation and applications," IEEE Transactions on Power Electronics, vol. 30(8), pp. 4091-4106, Aug 2015.

[8] X. Zhang and W. Yue, "Neutral point potential balance algorithm for three-level NPC inverter based on SHEPWM," Electronics Letters, vol. 53(23), pp. 1542-1544, Nov 2017.

[9] Y. Zhang, Z. Zhao and J. Zhu, "A hybrid PWM applied to high-power three-level inverter-fed induction-motor drives," IEEE Transactions on Industrial Electronics, vol. 58(8), pp. 3409-3420, Aug 2011.

[10] J. Wang and D. Ahmadi, "A precise and practical harmonic elimination method for multilevel inverters," IEEE Transactions on Industry Applications, vol. 46(2), pp. 857-865, Mar-Apr 2010.

[11] D. Ahmadi, K. Zou, C. Li, Y. Huang and J. Wang, "A universal selective harmonic elimination method for highpower inverters," IEEE Transactions on Power Electronics, vol. 26(10), pp. 2743-2752, Oct 2011.

[12] A. Edpuganti and A. K. Rathore, "A survey of low switching frequency modulation techniques for medium-voltage multilevel converters," IEEE Transactions on Industry Applications, vol. 51(5), pp. 4212-4228, Sept-Oct 2015.

[13] W. Zhang, X. Meng and Z. Li, "The simulation research for selective harmonic elimination technique based on genetic algorithm," Proceedings of the 33rd Chinese Control Conference, Nanjing, pp. 8628-8632, 2014.

[14] H. Taghizadeh and M. Tarafdar Hagh, "Harmonic elimination of cascade multilevel inverters with nonequal DC sources using particle swarm optimization," IEEE Transactions on Industrial Electronics, vol. 57(11), pp. 36783684, Nov 2010

[15] M. H. Etesami, N. Farokhnia and S. Hamid Fathi, "Colonial competitive algorithm development toward harmonic minimization in multilevel inverters," IEEE Transactions on Industrial Informatics, vol. 11(2), pp. 459-466, Apr 2015.

[16] A. Kavousi, B. Vahidi, R. Salehi, M. K. Bakhshizadeh, N. Farokhnia and S. H. Fathi, "Application of the bee algorithm for selective harmonic elimination strategy in multilevel inverters," IEEE Transactions on Power Electronics, vol. 27(4), pp. 1689-1696, Apr 2012.

[17] Chunhui $\mathrm{Wu}$, Qirong Jiang and Chunpeng Zhang, "An optimization method for three-level selective harmonic eliminated pulse width modulation (SHEPWM)," in International Conference on Electrical Machines and Systems, Nanjing, vol. 2, pp. 1346-1350, 2005.

[18] Y. Wang, X. Wen, X. Guo, F. Zhao and W. Cong, "The smooth transition research of different PWM modulations for vector control of induction motor in medium voltage high power," in International Conference on Electrical Machines and Systems, pp. 1-5, Beijing 2011.

[19] A. A. Radionov, V. R. Gasiyarov and A. S. Maklakov, "Hybrid PWM on the basis of SVPWM and SHEPWM for VSI as part of 3L-BtB-NPC converter," in IECON 2017 - 43rd Annual Conference of the IEEE Industrial Electronics Society, pp. 1232-1236, Beijing 2017. 
[20] Y. Zhang, D. Xu, C. Yan and S. Zou, "Hybrid PWM Scheme for the Grid Inverter, "IEEE Journal of Emerging and Selected Topics in Power Electronics, vol. 3(4), pp. 1151-1159, Dec 2015.

\section{BIOGRAPHIES OF AUTHORS}
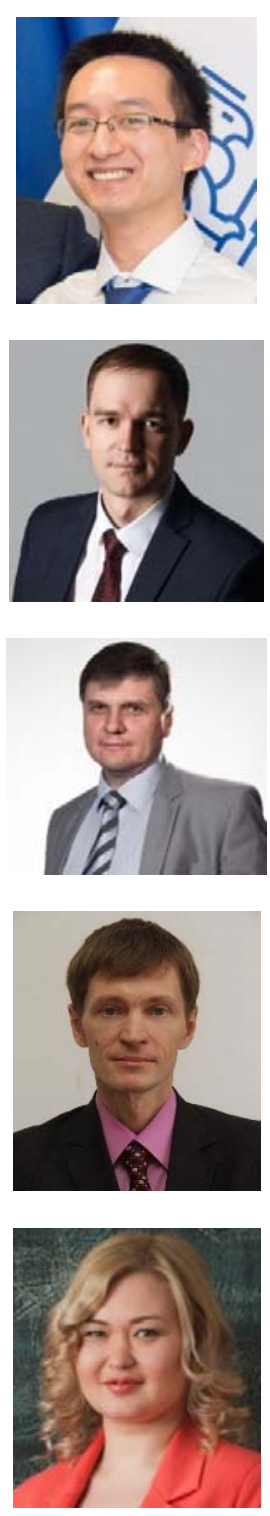

Tao Jing. 2016 - Diploma of a Bachelor, Zhejiang Ocean University, China; 2018 - Diploma of a Master, South Ural State University, Russia; 2018 to present - Doctoral candidate of the Department "Mechatronics and automation", South Ural State University, Russia. His research interests include the field of power electronics, motor drive systems and mechatronics.

Alexander Maklakov. 2013 - Diploma of an engineer, Magnitogorsk State Technical University, Russia; 2017 - PhD (technical science), South Ural State University, Russia; 2015 to present - Associate professor of the Department "Mechatronics and automation", South Ural State University, Russia. His research interests include the field of power electronics and motor drive systems.

Andrey Radionov. 1997 - Diploma of an engineer, Magnitogorsk State Technical University, Russia; 2000 - PhD (technical science), Moscow State Technical University (MEI), Russia; 2009 - Dr. Sc.(techn.), Magnitogorsk State Technical University, Russia; 2014 to present Vice-Rector for Education Affairs, South Ural State University, Russia. His research interests include the field of power electronics, motor drive systems and mechatronics.

Sergei Baskov. 1996 - Diploma of an engineer, Magnitogorsk State Technical University, Russia; 1999 - PhD (technical science), Moscow State Technical University (MEI), Russia; 2017 to present - Associate professor of the Department "Mechatronics and automation", South Ural State University, Russia. His research interests include the field of power electronics and motor drive systems.

Aleksandra Kulmukhametova. 2008 - Diploma of an engineer, South Ural State University, Russia; 2014 - PhD (technical science), Ufa state aviation technical university (USATU), Russia; 2014 to present - Associate professor of the Department "Mechatronics and automation", South Ural State University, Russia. His research interests include the field of motor drive systems. 